\title{
Analysis of Properties of Chosen Acoustic Emission Descriptors Describing Acoustic Signals Measured by Means of Acoustic Emission Method within Oil Transformers
}

\author{
F. Witos* AND A. Olszewska
}

Department of Optoelectronics, Silesian University of Technology, Krzywoustego 2, 44-100 Gliwice, Poland

Authors of the paper present investigation results concerning properties of ADP, ADC and ADNC descriptors which have been obtained during measurements made within three oil transformers and then tested also by other methods (electric and dissolved gas analysis ones). Methodology which makes easier an analysis and identification of acoustic emission signals generated by partial discharges is presented. Results obtained by acoustic emission method have been referred to results coming from other measuring methods.

PACS numbers: 52.80.--s, 77.22.Jp, 84.70.+p

\section{Introduction}

Acoustic emission methods belong to non-electric methods applied to investigate the partial discharges (PD). They create a unique possibility to observe deformation processes as well as locate and identify the sources of PD.

In the hereby work an author's method [1] of registration and analysis of acoustic emission (AE) signals generated by partial discharges is used. Such signals have come from three oil power transformers in which partial discharges have appeared.

Analysis of AE signals is composed of two stages - basic and advanced description. In the frame of the basic analysis of data the "whole" AE signals whose duration is $2 \mathrm{~s}$ (100 periods of the supply voltage) are analyzed in domain of the time, frequency and time-frequency. An example description of AE signal is presented in Figs. 3 and 4. Advanced analysis of data enlarges a preliminary description of signals. This work is concentrated on advanced analysis of signals in threshold domain that includes calculation of amplitude distributions (counting tempo, power of a signal and counting rate) as well as specially defined AE descriptors - ADP, ADC and ADNC [1-4]. Such descriptors describe an advanced degree of recorded $\mathrm{AE}$ signals which is connected with the advanced degree of analyzed deformation process.

The purpose of the present work was the carrying out of analysis of properties of descriptors $\mathrm{ADP}, \mathrm{ADC}$ and $\mathrm{ADNC}$ for signals recorded by means of $\mathrm{AE}$ method at chosen measuring points distributed on transformer tanks and then application of these descriptors to selection of advanced AE signals and location of PD sources.

\section{Subject and methodology of investigations}

As a subject of investigations three oil power transformers were used:

\footnotetext{
* corresponding author; e-mail: franciszek.witos@polsl.pl
}

- transformer $A$ (Fig. 1), power of 25 MVA, rated voltage of $110 \mathrm{kV}$,

- transformer $B$, power of $25 \mathrm{MVA}$, rated voltage of $110 \mathrm{kV}$,

- transformer $C$, power of 16 MVA, rated voltage of $110 \mathrm{kV}$.

Results of several last tests of the oil coming from transformers $B$ and $C$, made by means of chromatograph dissolved gas analysis (DGA), proved an increased level of hydrogen and other gases. It showed that there are partial discharges within these transformers. Level of gases within transformer $A$ was situated in the range acceptable by suitable standards.

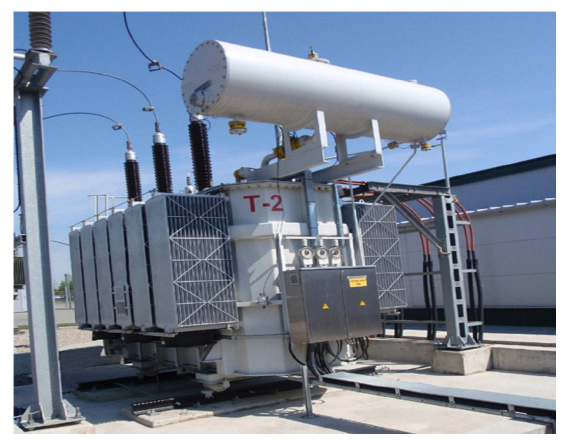

Fig. 1. Photograph of transformer $A$.

Investigations of partial discharges within transformers $A$ and $B$ have been made on-line by means of $\mathrm{AE}$ method whereas transformer $C$ was tested in the firm Power Engineering in Czerwonak.

In order to investigate transformers an author's measuring system called AE DEMA-COMP [1-4] was applied; such a system has been equipped with AE sensors R6 type, produced by Physical Acoustic Corporation. AE signals have been recorded at chosen accessible points of the lateral surface of transformer tank. Such a recording lasted $2 \mathrm{~s}$ (100 periods of the supply voltage). 


\section{Analysis of properties of ADP, ADC and ADNC descriptors}

At first, signals recorded by means of AE method have been put to filtration; band of filtration is done as a range of frequency at frequency characteristics. Subsequently, for each of signal the following quantities have been calculated: basic characteristics, amplitude distributions of signals and corresponding with them amplitude descriptors of: power of signal (ADP), count of signal(ADC), and normalized count of signal (ADNC).

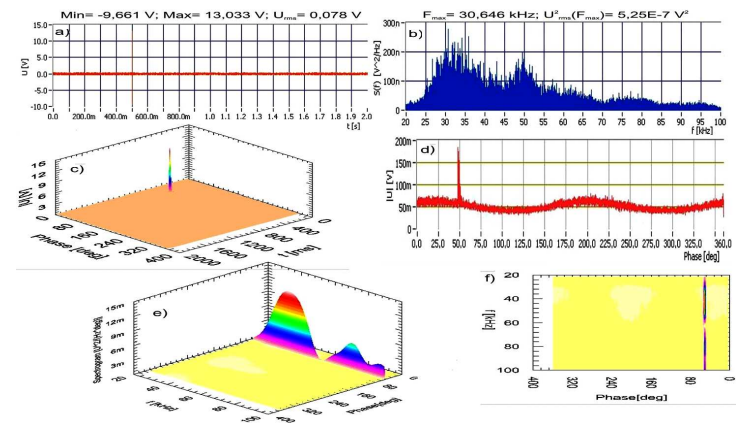

Fig. 2. Preliminary description of AE signal with interference (T1E12.BIN): (a) signal, (b) frequency characteristic, (c) phase-time characteristic, (d) averaging phase characteristic, (e) and (f) averaging spectrograms $\mathrm{STFT} ; \mathrm{ADP}=-0.03, \mathrm{ADC}=-0.36, \mathrm{ADNC}=-0.37$.

Calculated descriptors can be easily ranged by their values. Searching for PD sources an analysis may narrow to signals for which descriptors have great values. However, for such signals it is necessary to verify their periodic character. Periodicity of signals is well visible particularly at phase-time characteristics where are well visible non-periodic events which can be external disturbances or acoustic noises $[1,5]$. If such events appeared they can be easily eliminated. Figures 2 and 3 present basic characteristics of AE signal with interferences and characteristics after removing of these interferences.

After rejection of single impulse at phase-time characteristic ADP, ADC, and ADNC descriptor values decrease considerably. Their values for both cases have been presented under the figures. Indication for procedure of analysis of AE signals is additional security in order to eliminate effects which are not connected with
$\mathrm{PD}$ resultant from AE signals.

After carrying out this procedure, the following parameters of ADP, ADC, and ADNC descriptors for signals recorded at each measuring point have been calculated: mean value $a$, correlation coefficient $r$, average standard deviation $\sigma$ and proportional uncertainty $\Delta$.

Calculated parameters resultant from advanced analysis of AE signals recorded at chosen measuring points of transformers are presented in Tables I-III. There are mean values $a$, correlation coefficient $r$, average standard deviation $\sigma$ and proportional uncertainty $\Delta$ for ADP, $\mathrm{ADC}$ and ADNC descriptors. Arrangement according to descriptor values is made in Tables I, II whereas in Table III they are arranged according to signal groups - in accordance with their location in transformer and their properties.

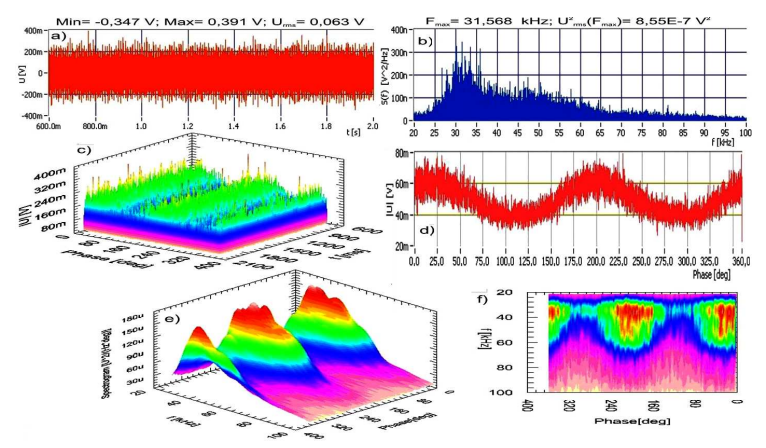

Fig. 3. Preliminary description of AE signal after interference elimination (T1E12.BIN): (a) signal, (b) frequency characteristic, (c) phase-time characteristic, (d) averaging phase characteristic, (e) and (f) averaging spectrograms STFT; $\mathrm{ADP}=-13.91, \mathrm{ADC}=-15.26$, $\mathrm{ADNC}=-16.54$

Descriptor values for transformer $A$ are small since their greatest values are as follows: $\mathrm{ADP}=-8.93$, $\mathrm{ADC}=-11.34, \mathrm{ADNC}=-12.33$. Approximation of descriptors is very good because values of coefficient correlation $r$ are situated within an interval [1.00; 0.98]. Proportional relative uncertainties of descriptors are of several percents. Exemplary basic description of a signal is presented in Fig. 4. Because investigations made by means of DGA method show that there are no PD within this transformer, so presented results (Table I, Fig. 4) describe acoustic noise of working transformer.

TABLE I

Selected parameters of advanced analysis of AE signals recorded at chosen measuring points of transformer $A$.

\begin{tabular}{c|c|c|c|c|c|c|c|c|c|c|c|c|c}
\hline \hline \multirow{2}{*}{ No. } & \multirow{2}{*}{ Name } & \multicolumn{4}{|c|}{ ADP } & \multicolumn{4}{c|}{ ADC } & \multicolumn{4}{c}{ ADNC } \\
\cline { 3 - 13 } & & $a$ & $r$ & $\sigma$ & $\Delta[\%]$ & $a$ & $r$ & $\sigma$ & $\Delta[\%]$ & $a$ & $r$ & $\sigma$ & $\Delta[\%]$ \\
\hline 1 & TR2M04 & -8.93 & 0.99 & 0.30 & 3.35 & -11.34 & 0.99 & 0.32 & 2.86 & -12.33 & 0.99 & 0.31 & 2.54 \\
2 & TR2P06 & -12.22 & 1.00 & 0.48 & 3.93 & -13.63 & 1.00 & 0.35 & 2.55 & -15.25 & 1.00 & 0.43 & 2.84 \\
3 & TR2R06 & -16.99 & 0.99 & 0.77 & 4.55 & -17.36 & 0.99 & 0.58 & 3.31 & -19.33 & 0.99 & 0.60 & 3.11 \\
4 & TR2M03 & -20.53 & 0.99 & 0.33 & 1.62 & -19.04 & 0.98 & 0.19 & 1.01 & -21.71 & 0.99 & 0.31 & 1.44 \\
5 & TR2P02 & -22.61 & 0.99 & 1.12 & 4.96 & -20.91 & 0.98 & 0.56 & 2.69 & -23.47 & 0.98 & 0.77 & 3.28
\end{tabular}


TABLE II

Selected parameters of advanced analysis of AE signals recorded at chosen measuring points of transformer $B$.

\begin{tabular}{c|c|c|c|c|c|c|c|c|c|c|c|c|c}
\hline \hline \multirow{2}{*}{ No. } & \multirow{3}{*}{ Name } & \multicolumn{3}{|c|}{ ADP } & \multicolumn{4}{c|}{ ADC } & \multicolumn{4}{c}{ ADNC } \\
\cline { 3 - 13 } & & $a$ & $r$ & $\sigma$ & $\begin{array}{c}\Delta \\
{[\%]}\end{array}$ & $a$ & $r$ & $\sigma$ & $\begin{array}{c}\Delta \\
{[\%]}\end{array}$ & $a$ & $r$ & $\sigma$ & $\begin{array}{c}\Delta \\
{[\%]}\end{array}$ \\
\hline 1 & T1B04 & -0.86 & 0.99 & 0.04 & 4.95 & -1.28 & 0.99 & 0.03 & 2.64 & -1.43 & 0.99 & 0.04 & 3.06 \\
2 & T1G01 & -1.27 & 0.99 & 0.06 & 4.48 & -1.80 & 0.99 & 0.04 & 2.19 & -2.03 & 0.99 & 0.05 & 2.55 \\
3 & T1I03 & -1.91 & 0.99 & 0.14 & 7.13 & -2.59 & 0.97 & 0.17 & 6.64 & -3.01 & 0.98 & 0.18 & 6.13 \\
4 & T1H02 & -2.50 & 0.99 & 0.15 & 6.16 & -3.09 & 0.99 & 0.14 & 4.41 & -3.48 & 0.99 & 0.16 & 4.49 \\
5 & T1B03 & -4.55 & 0.99 & 0.36 & 7.81 & -5.81 & 0.99 & 0.35 & 5.98 & -6.35 & 0.99 & 0.36 & 5.62 \\
6 & T1B05 & -6.48 & 0.99 & 0.54 & 8.39 & -7.29 & 1.00 & 0.32 & 4.35 & -7.93 & 0.99 & 0.43 & 5.46 \\
7 & T1D02 & -10.17 & 0.99 & 0.24 & 2.34 & -10.57 & 0.99 & 0.13 & 1.23 & -11.13 & 0.99 & 0.14 & 1.29 \\
8 & T1105 & -11.18 & 0.98 & 0.30 & 2.72 & -11.38 & 0.98 & 0.19 & 1.67 & -11.65 & 0.98 & 0.24 & 2.08 \\
9 & T1D09 & -12.71 & 0.99 & 0.20 & 1.58 & -12.87 & 0.99 & 0.13 & 1.01 & -13.27 & 0.99 & 0.15 & 1.11 \\
10 & T1E10 & -15.15 & 0.99 & 0.49 & 3.23 & -16.55 & 0.99 & 0.24 & 1.43 & -17.09 & 0.99 & 0.44 & 2.56
\end{tabular}

TABLE III

Selected parameters of advanced analysis of AE signals recorded at chosen measuring points of transformer $C$.

\begin{tabular}{|c|c|c|c|c|c|c|c|c|c|c|c|c|c|}
\hline \multirow[b]{2}{*}{ No. } & \multirow[b]{2}{*}{ Name } & \multicolumn{4}{|c|}{$\mathrm{ADP}$} & \multicolumn{4}{|c|}{$\mathrm{ADC}$} & \multicolumn{4}{|c|}{ ADNC } \\
\hline & & $a$ & $r$ & $\sigma$ & $\begin{array}{c}\Delta \\
{[\%]}\end{array}$ & $a$ & $r$ & $\sigma$ & $\begin{array}{c}\Delta \\
{[\%]}\end{array}$ & $a$ & $r$ & $\sigma$ & $\begin{array}{c}\Delta \\
{[\%]}\end{array}$ \\
\hline 1 & C191 & -3.00 & 0.95 & 0.87 & 29.18 & -4.35 & 0.92 & 1.16 & 26.72 & -5.02 & 0.95 & 1.26 & 25.07 \\
\hline 2 & C192 & -0.52 & 0.93 & 0.15 & 29.00 & -0.91 & 0.86 & 0.17 & 18.24 & -1.13 & 0.91 & 0.21 & 18.87 \\
\hline 3 & C193 & -1.41 & 0.92 & 0.05 & 3.53 & -1.98 & 0.93 & 0.04 & 1.94 & -2.42 & 0.95 & 0.04 & 1.51 \\
\hline 4 & C194 & -1.77 & 0.94 & 0.15 & 8.44 & -2.61 & 0.92 & 0.15 & 5.59 & -3.14 & 0.94 & 0.19 & 5.95 \\
\hline 5 & C201 & -0.79 & 0.99 & 0.07 & 8.90 & -1.44 & 0.94 & 0.04 & 2.46 & -1.62 & 0.95 & 0.05 & 3.11 \\
\hline 6 & C202 & -2.88 & 0.96 & 0.63 & 21.86 & -4.65 & 0.91 & 0.85 & 18.25 & -5.19 & 0.93 & 0.94 & 18.11 \\
\hline 7 & C203 & -3.05 & 0.95 & 0.80 & 26.35 & -4.53 & 0.91 & 1.03 & 22.61 & -5.25 & 0.94 & 1.12 & 21.25 \\
\hline 8 & C204 & -8.17 & 1.00 & 0.47 & 5.77 & -9.41 & 0.99 & 0.47 & 4.95 & -10.69 & 1.00 & 0.48 & 4.46 \\
\hline 9 & C61 & -4.00 & 0.97 & 0.45 & 11.28 & -5.79 & 0.95 & 0.58 & 10.00 & -6.47 & 0.97 & 0.64 & 9.91 \\
\hline 10 & C62 & -2.94 & 0.97 & 0.21 & 7.01 & -4.34 & 0.94 & 0.27 & 6.23 & -5.06 & 0.96 & 0.27 & 5.43 \\
\hline 11 & C63 & -4.19 & 0.99 & 0.30 & 7.12 & -5.31 & 0.98 & 0.32 & 5.98 & -6.26 & 0.99 & 0.37 & 5.86 \\
\hline 12 & C161 & -4.34 & 0.95 & 0.91 & 21.01 & -6.01 & 0.93 & 0.99 & 16.47 & -6.79 & 0.95 & 1.12 & 16.45 \\
\hline 13 & C162 & -4.74 & 0.94 & 1.04 & 21.88 & -6.59 & 0.92 & 1.12 & 17.00 & -7.55 & 0.94 & 1.26 & 16.71 \\
\hline 14 & C163 & -5.21 & 0.97 & 1.01 & 19.47 & -7.16 & 0.95 & 1.24 & 17.29 & -8.05 & 0.96 & 1.32 & 16.46 \\
\hline 15 & C164 & -6.35 & 0.98 & 0.56 & 8.84 & -8.09 & 0.97 & 0.60 & 7.45 & -9.22 & 0.98 & 0.62 & 6.70 \\
\hline 16 & C100 & -11.40 & 0.99 & 0.57 & 5.03 & -12.62 & 0.99 & 0.48 & 3.79 & -13.73 & 0.99 & 0.47 & 3.45 \\
\hline 17 & C102 & -12.79 & 1.00 & 0.49 & 3.87 & -14.03 & 0.99 & 0.27 & 1.95 & -16.07 & 1.00 & 0.37 & 2.28 \\
\hline 18 & C111 & -12.24 & 0.99 & 1.28 & 10.48 & -13.92 & 0.98 & 1.28 & 9.20 & -14.95 & 0.99 & 1.07 & 7.16 \\
\hline 19 & C112 & -12.69 & 1.00 & 0.71 & 5.63 & -14.25 & 0.99 & 0.63 & 4.41 & -15.77 & 1.00 & 0.60 & 3.80 \\
\hline 20 & C113 & -12.34 & 1.00 & 0.64 & 5.15 & -14.18 & 0.99 & 0.69 & 4.86 & -15.13 & 1.00 & 0.54 & 3.54 \\
\hline 21 & C114 & -12.17 & 0.99 & 0.96 & 7.89 & -13.54 & 0.99 & 0.87 & 6.46 & -14.67 & 1.00 & 0.79 & 5.38 \\
\hline
\end{tabular}

Majority of AE signals coming from transformer $B$ have properties characteristic for acoustic noise during its work - signals $7-10$ in Table II. There are also signals with different properties - see 1-6 in Table II; their common feature is appearance of higher values of descriptors. They can be divided into two groups. Signals from the first group (signals 1-3 in Table II) have higher values of descriptors and lesser proportional relative uncertainties of descriptors; exemplary basic description of AE signal from such a group is in Fig. 5. Signals from the second group (signals $4-6$ in Table II) have lesser values of descriptors and higher proportional relative uncertainties 
of descriptors; exemplary basic description of AE signal from such a group is in Fig. 6 .

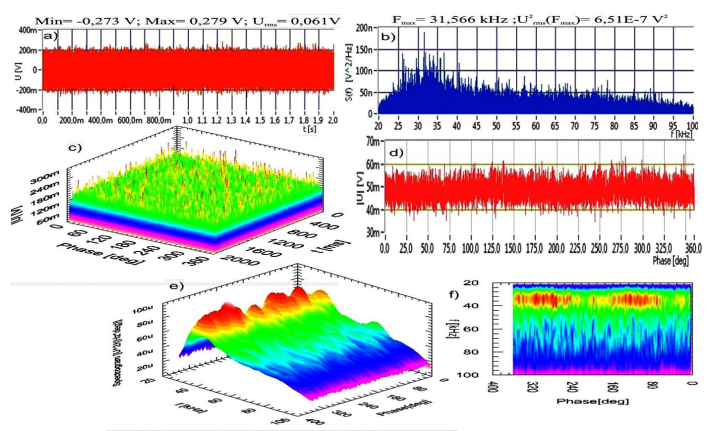

Fig. 4. Basic description of AE signal recorded at chosen measuring point of transformer $A$ (source TR2MO3(3).BIN): (a) signal, (b) frequency characteristic, (c) phase-time characteristic, (d) averaging phase characteristic, (e) and (f) averaging spectrograms STFT; $\mathrm{ADP}=-20.53$.

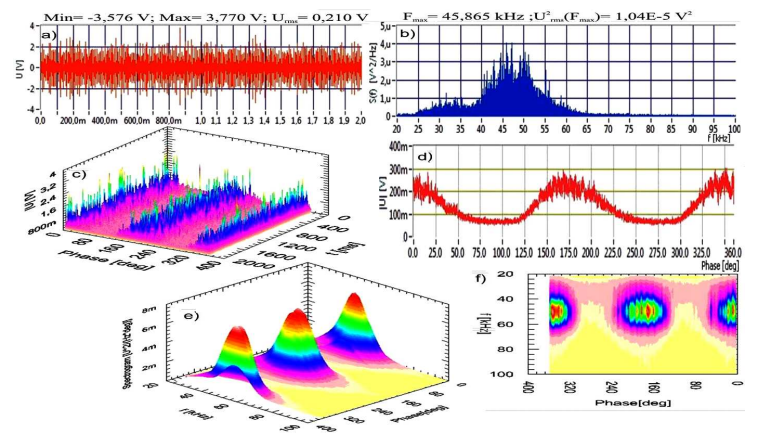

Fig. 5. Basic description of AE signal recorded at chosen measuring point of transformer $B$ (source T1BO4(4).BIN): (a) signal, (b) frequency characteristic, (c) phase-time characteristic, (d) averaging phase characteristic, (e) and (f) averaging spectrograms STFT; $\mathrm{ADP}=-0.86$

Location of measuring points is not described in the present work but it is worth to mention that all points belonging to the first group are situated into two well located areas on the tank of transformer $B$ whereas points from the second group are situated near PD sources. Such information enables us to locate PD sources (signals from the first group) and interpret signals from the second group as signals with great amplitude coming from the same PD sources which are so great as they are not damped yet on the road of propagation.

It is worth to add that - according to our suggestion - the revision of transformer $B$ has been made. During of such a revision it turned out that conductors connecting HV leads and tap changer have been situated too near the transformer tank on pointed areas. Owing to such a reason Fig. 5 presents information about "outside" PD sources in a transformer.

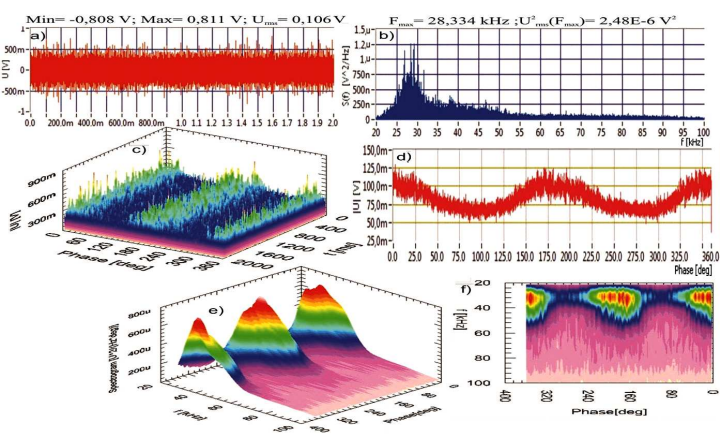

Fig. 6. Basic description of AE signal recorded at chosen measuring point of transformer $B$ (source T1BO3(2).BIN): (a) signal, (b) frequency characteristic, (c) phase-time characteristic, (d) averaging phase characteristic, (e) and (f) averaging spectrograms STFT; $\mathrm{ADP}=-4.55$

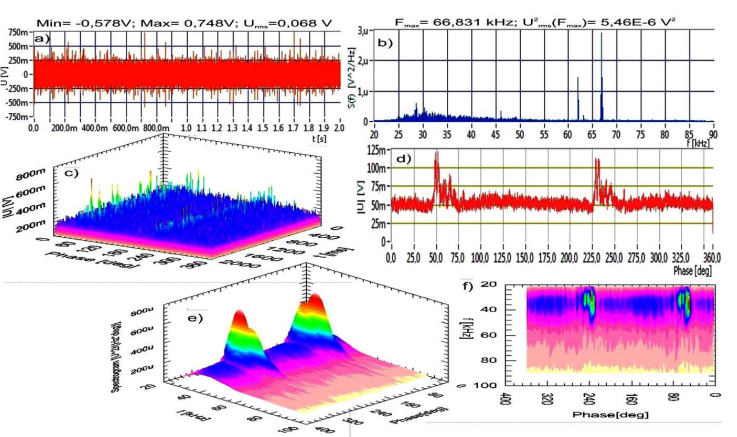

Fig. 7. Basic description of AE signal recorded at chosen measuring point of transformer $C$ (source C161(1).BIN): (a) signal, (b) frequency characteristic, (c) phase-time characteristic, (d) averaging phase characteristic, (e) and (f) averaging spectrograms STFT; $\mathrm{ADP}=-4.34$.

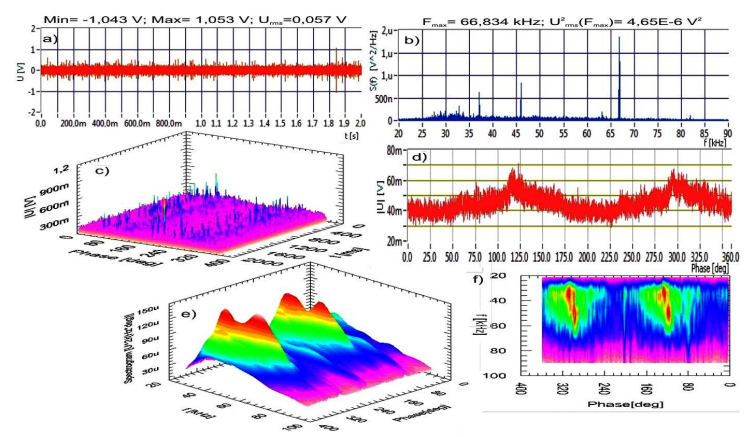

Fig. 8. Basic description of AE signal recorded at chosen measuring point of transformer $C$ (source C203(1).BIN): (a) signal, (b) frequency characteristic, (c) phase-time characteristic, (d) averaging phase characteristic, (e) and (f) averaging spectrograms STFT; $\mathrm{ADP}=-3.05$. 
Signals within transformer $C$ have the most complex character. Descriptor values at majority of measuring points are considerably higher than in the case of distributions for remaining transformers. This means that signals describe deformation processes with higher advance degree. Descriptors ADP, ADC and ADNC take great values; their fluctuation is also greater. In the group of great values of descriptors different dependences and level of fluctuation are observed. Several groups can be separated. By comparison with preceding transforms there is a group of signals with stable value of descriptors within the group but with great fluctuations at the point; correlation coefficient $r$ for many of these signals is in the range of 0.86-1.00. This worse fitting describes more complex character of amplitude distributions. Exemplary basic description of AE signal from such a group is presented in Fig. 7. It is possible to separate groups with great fluctuations of descriptor values in the group. Exemplary basic description of AE signal from such a group is presented in Fig. 8. Detailed analysis of AE signals for this transformer is a contents of another article because - parallel to investigations carried out by means of AE method there are tests made by means of electric method which proved appearance of excessively great apparent charge on the level of $30 \mathrm{nC}$.

\section{Recapitulation}

AE method has been applied on-line for oil power transformers. Specially designed enclosures of AE sensors enable us to measure PD at points of a transformer tank also between its radiators [6-12].

Owing to ADP, ADC, and ADNC descriptors the quantitative estimation of advanced degree of recorded signals was obtained.

Examination of two transformers with identical construction but different exploitation parameters enables us to qualification of acoustic noise of a working transformer (Fig. 3), discrimination of signals coming from PD and approximate location of PD sources. Revision of transformer $B$ proved correctness of such a location and determined the kind of PD source: a discharge in the oil between conductors connecting HV leads with a tap changer and transformer tank. Revision results proved that AE coming from such a source is measurable; Fig. 5 gives information about properties of AE signals coming from "outside" PD sources in a transformer.

There is an open question to degree of measurability of signals coming from PD sources situated inside a transformer. Considerable variety of $\mathrm{AE}$ signals and great wealth of advanced parameters enumerated in Table III allows us to suppose that it will be possible in many cases. However, the further investigations are necessary to obtain detailed responses.

Additional verification of periodical character of recorded signals has been made in the case of signals with values of descriptors; it is additionally security in order to eliminate effects whose are not connected with partial discharges recorded with AE signals.

\section{References}

[1] F. Witos, Investigation of Partial Discharges by Means of Acoustic Emission Method and Electric Method, Wyd. Politechniki Śląskiej, Gliwice 2008 (in Polish).

[2] F. Witos, Z. Gacek, A. Opilski, Arch. Acoust. 27, 65 (2002).

[3] F. Witos, Z. Gacek, Europ. Phys. J.-Spec. Top. 154, 239 (2008).

[4] F. Witos, Z. Gacek, Acta Phys. Pol. A 116, 422 (2009).

[5] F. Witos, Z. Gacek, Energetyka 6, 363 (2009).

[6] M. MacAlpine, Z. Zhiquiang, M.S. Demokan, Electr. Power Syst. Res. 63, 27 (2002).

[7] J. Deng, H. Xiao, W. Huo, M. Luo, R. May, A. Wang, Y. Liu, Opt. Laser Technol. 33, 305 (2001).

[8] A.S. Faraq, M.H. Shewdi, X. Jin, C. Wang, T.C. Cheng, X. Dong, S. Gao, W. Jing, Z. Wang, Electr. Power Syst. Res. 50, 47 (1999).

[9] Z. Deheng, T. Kexiong, J. Xianche, in: II Conf. Properties and Application of Dielectric Materials, Ed. L. Zao, Vol. 2, University of Bengin, Bejing 1988, p. 614.

[10] F. Witos, Z. Gacek, Z. Opilski, Acta Phys. Pol. A 114, A-247 (2008).

[11] T. Boczar, S. Borucki, A. Cichon, M. Lorenc, Acta Phys. Pol. A, 114, A-19 (2008).

[12] T. Boczar, P. Frącz, Acta Phys. Pol. A 116, 281 (2009). 